\title{
Virasoro constraints and flavor-topology duality in QCD
}

\author{
D. Dalmazi* \\ UNESP, Guaratinguetá, S.P., 12.500.000, Brazil \\ J. J. M. Verbaarschot ${ }^{\dagger}$ \\ Department of Physics and Astronomy, SUNY, Stony Brook, New York 11794
}

(Received 8 January 2001; published 31 July 2001)

\begin{abstract}
We derive Virasoro constraints for the zero momentum part of the QCD-like partition functions in the sector of topological charge $\nu$. The constraints depend on the topological charge only through the combination $N_{f}$ $+\beta \nu / 2$ where the value of the Dyson index $\beta$ is determined by the reality type of the fermions. This duality between flavor and topology is inherited by the small-mass expansion of the partition function and all spectral sum rules of inverse powers of the eigenvalues of the Dirac operator. For the special case $\beta=2$ but arbitrary topological charge the Virasoro constraints are solved uniquely by a generalized Kontsevich model with the potential $\mathcal{V}(X)=1 / X$.
\end{abstract}

DOI: 10.1103/PhysRevD.64.054002

\section{FLAVOR-TOPOLOGY DUALITY}

Through the work of 't Hooft we know that the lowenergy limit of QCD is dominated by light flavors and topology [1]. We expect that the same will be the case for the low-lying eigenvalues of the Dirac operator. Indeed, for massless flavors, the fermion determinant results in the repulsion of eigenvalues away from $\lambda=0$. It is perhaps less known that the presence of exactly zero eigenvalues has the same effect. The reason is the repulsion of eigenvalues which occurs in all interacting systems and has probably best been understood in the context of random matrix theory where the eigenvalues obey the Wigner repulsion law [2].

In QCD, the fluctuations of the low-lying eigenvalues of the Dirac operator are described by chiral random matrix theory (chRMT) [3-5]. This is a random matrix theory with the global symmetries of the QCD partition function. It is characterized by the Dyson index [6] $\beta$ which is equal to the number of independent variables per matrix element. For QCD with fundamental fermions we have $\beta=1$ for $N_{c}=2$ and $\beta=2$ for $N_{c}>2$. For QCD with adjoint fermions and $N_{c} \geqslant 2$ the Dirac matrix can be represented in terms of selfdual quaternions with $\beta=4$. The main ingredient of the chRMT partition function is the integration measure which includes the Vandermonde determinant. In terms of Dirac eigenvalues $i \lambda_{k}$ it is given by $\Pi_{k<l}\left|\lambda_{k}^{2}-\lambda_{l}^{2}\right|^{\beta} \lambda_{k}^{\beta \nu+\beta-1}$. Therefore, the presence of $N_{f}$ massless flavors, with the fermion determinant given by $\Pi_{k} \lambda_{k}^{2 N_{f}}$, has the same effect on eigenvalue correlations as $\nu=2 N_{f} / \beta$ zero eigenvalues. More precisely, the joint eigenvalue distribution only depends on the combination $2 N_{f}+\beta \nu[4,7]$. Based on the conjecture $[3,4]$ that the zero-momentum part of the QCD partition function, $Z_{N_{f}, \nu}\left(m_{1}, \ldots, m_{N_{f}}\right)$, is a chiral random matrix theory, it was suggested [8] that its mass dependence obeys the duality relation (for $\beta=2$ )

\footnotetext{
*Email address: dalmazi@feg.unesp.br

${ }^{\dagger}$ Email address: verbaarschot@nuclear.physics.sunysb.edu
}

PACS number(s): 12.39.Fe, 11.30.Rd, 12.38.Lg

$$
\begin{aligned}
& Z_{N_{f}, \nu}\left(m_{1}, \ldots, m_{N_{f}}\right) \\
& \quad \sim \prod_{f} m_{f}^{\nu} Z_{N_{f}+\nu, 0}\left(m_{1}, \ldots, m_{N_{f}}, 0, \ldots, 0\right) .
\end{aligned}
$$

This relation, which is now known as flavor topologyduality, is a trivial consequence of the flavor dependence of the chRMT joint eigenvalue distribution.

The mass dependence of the chRMT partition function can be reduced to a unitary matrix integral which is known from the zero momentum limit of chiral perturbation theory $[9,10]$. Starting from this representation of the low energy limit of the QCD partition function, also known as the finite volume partition function, flavor-topology duality was first proved for $N_{f}=2$ in [8]. However, its generalization to arbitrary $N_{f}$ and $\nu$ has only been achieved for $\beta=2[11,12]$. The relation (1) has been particularly useful for establishing relations between correlation functions of Dirac eigenvalues and finite volume partition functions [12-16].

For $\beta=2$ the unitary matrix integral, which represents the low-energy limit of the QCD partition function, is also known as the one-link integral of two-dimensional QCD (see for example [17]) or the Brezin-Gross-Witten model [18]. For zero topological charge it can also be represented as a generalized Kontsevich model [19] with potential $\mathcal{V}(X)$ $=1 / X$. This model has been discussed extensively in the context of topological gravity (for reviews see [23-25]). In this case the one-link integral has been analyzed $[19,20]$ by means of Virasoro constraints which are based on its invariance properties. The main issue we wish to address in this article is whether flavor-topology duality of the one-link integral can be understood without relying on its chRMT representation. We will do this by deriving Virasoro constraints for arbitrary topological charge. For $\beta=2$ we find a nonperturbative solution of these constraints in the form of a generalized Kontsevich model.

In Sec. II we will derive the Virasoro constraints for arbitrary $\nu$ and three different values of $\beta$. We observe that they satisfy the flavor-topology duality relations. A recursive solution of these relations is presented in Sec. III. It also provides us with an efficient derivation of sum rules for the 
inverse Dirac eigenvalues to high order. In the second part of this section we discuss the uniqueness of the nonperturbative solution of the Virasoro constraints for $\beta=2$. Concluding remarks are made in Sec. IV.

\section{VIRASORO CONSTRAINTS}

In the simplest case, $\beta=2$ and vanishing topological charge $\nu=0$, the small-mass Virasoro constraints were first found in [19] after an identification of the appropriate unitary integral and the corresponding generalized Kontsevich model (GKM). Here we work entirely in the context of unitary integrals and derive a simple form of the Virasoro constraints valid for arbitrary topological charge and $\beta$. They are obtained by expanding the partition function in powers of the masses.

\section{A. $\boldsymbol{\beta}=\mathbf{2}$}

The low energy limit of QCD in the phase of broken chiral symmetry is a gas of weakly interacting Goldstone bosons. Its partition function is determined uniquely by the invariance properties of the Goldstone fields. For quark masses $m$ and space-time volume $V$ in the range

$$
\frac{1}{\Lambda} \ll V^{1 / 4} \ll \frac{1}{m_{\pi}}
$$

(where $m_{\pi}=\sqrt{2 m \Sigma / F^{2}}$ is the mass of the Goldstone modes with $F$ the pion decay constant and $\Sigma$ the chiral condensate) the partition function for the Goldstone modes factorizes $[9,10]$ into a zero-momentum part, also known as the finite volume partition function, and a nonzero momentum part. For fundamental fermions and a gauge group with $N_{c} \geqslant 3$, the finite volume partition function in the sector of topological charge $\nu$ is given by the following integral over the unitary group $[9,10]$

$$
\mathcal{Z}_{\nu}^{\beta=2}\left(\mathcal{M}, \mathcal{M}^{\dagger}\right)=\int_{U \in U\left(N_{f}\right)} d U(\operatorname{det} U)^{v} e^{(1 / 2) \operatorname{Tr}\left(\mathcal{M} U^{\dagger}+\mathcal{M}^{\dagger} U\right)},
$$

where $\mathcal{M}=M V \Sigma$. Here and below we always take $\nu \geqslant 0$. The quantity $M$ stands for the original unscaled quark mass matrix. The partition function is normalized such that $\mathcal{Z}_{v}^{\beta=2}\left(\mathcal{M}, \mathcal{M}^{\dagger}\right) \rightarrow \operatorname{det}^{\nu}(\mathcal{M})$ for $\mathcal{M} \rightarrow 0$.

Especially for $\nu=0$, the integral (3) has been studied extensively in the literature. In the context of lattice QCD it is known as the one-link integral $[18,21,19]$ (see also [17] for a review). Of particular interest is the fact that for $N_{f} \rightarrow \infty$ the partition function $\mathcal{Z}_{v}^{\beta=2}$ undergoes a phase transition [18] from a small mass phase $(\mathcal{M} \rightarrow 0)$ to a large mass phase $(\mathcal{M} \rightarrow \infty)$. The large mass expansion is asymptotic and its coefficients can be calculated, even for finite $N_{f}$, by expanding about a flavor-symmetric saddle point. $\operatorname{For} \operatorname{Re}(\mathcal{M}) \rightarrow \infty$ such expansion can be carried out in a efficient way by means of the (large-mass) Virasoro constraints found in [22]. For $\operatorname{Im}(\mathcal{M}) \rightarrow \infty$, at fixed value of $\operatorname{Re}(\mathcal{M})$, other flavornonsymmetric saddle points contribute to the partition function as well, and it is not known whether the expansion can be carried out by means of Virasoro constraints (for an explicit calculation including nonsymmetric saddle points see [26]). On the other hand, the small-mass expansion has a non-zero radius of convergence, and it can be determined by different methods for any finite $N_{f}$. For example, one can expand the exponential in Eq. (3) and calculate the corresponding unitary integrals systematically using a character expansion $[27,28]$. Another efficient technique is again the use of the (small-mass) Virasoro constraints found in $[19,20,12]$. By generalizing such constraints to arbitrary topological charge $\nu$ we will show that they naturally lead to flavor-topology duality.

First of all, using the unitary invariance of the measure in Eq. (3) we can deduce the covariance properties of the partition function under a redefinition of the mass matrix $\mathcal{M}$ $\rightarrow V^{-1} \mathcal{M} U$,

$$
\begin{aligned}
& (\operatorname{det} \mathcal{M})^{-\nu} \mathcal{Z}_{\nu}^{\beta=2}\left(\mathcal{M}, \mathcal{M}^{\dagger}\right) \\
& \quad=\left(\operatorname{det} V^{-1} \mathcal{M} U\right)^{-\nu} \mathcal{Z}_{\nu}^{\beta=2}\left(V^{-1} \mathcal{M} U, U^{-1} \mathcal{M}^{\dagger} V\right) .
\end{aligned}
$$

This equation implies that $(\operatorname{det} \mathcal{M})^{-\nu} \mathcal{Z}_{v}^{\beta=2}\left(\mathcal{M}, \mathcal{M}^{\dagger}\right)$ is a symmetric function of the eigenvalues of the Hermitian matrix $L_{a b} \equiv\left(\mathcal{M M}^{\dagger}\right)_{a b}$. Following [19] we can introduce the infinite set of variables

$$
t_{k}=\frac{1}{k} \operatorname{Tr}\left(\frac{\mathcal{M} \mathcal{M}^{\dagger}}{4}\right)^{k}, \quad k \geqslant 1,
$$

which are explicitly symmetric with respect to permutations of the eigenvalues and write

$$
(\operatorname{det} \mathcal{M})^{-\nu} \mathcal{Z}_{\nu}^{\beta=2}\left(\mathcal{M}, \mathcal{M}^{\dagger}\right)=G_{\nu}\left(t_{k}\right),
$$

where $G_{\nu}\left(t_{k}\right)$ has the Taylor expansion

$$
G_{\nu}\left(t_{k}\right)=1+a_{1} t_{1}+a_{2} t_{2}+a_{11} t_{1}^{2}+\cdots .
$$

A simple consistency check on Eq. (6) is that unitary integrals are only nonvanishing if the powers of $U$ and $U^{\dagger}$ are equal. Following [19] we can find the coefficients of the Taylor expansion (7) from the differential equation

$$
\frac{\partial^{2} \mathcal{Z}_{\nu}^{\beta=2}}{\partial \mathcal{M}_{b a} \partial \mathcal{M}_{a c}^{\dagger}}=\frac{\delta_{c b}}{4} \mathcal{Z}_{\nu}^{\beta=2}
$$

which follows directly from Eq. (3). As a consequence, $G_{\nu}\left(t_{k}\right)$ satisfies the equation

$$
\left[\frac{\partial^{2}}{\partial \mathcal{M}_{b a} \partial \mathcal{M}_{a c}^{\dagger}}+\nu \mathcal{M}_{a b}^{-1} \frac{\partial}{\partial \mathcal{M}_{a c}^{\dagger}}\right] G_{\nu}\left(t_{k}\right)=\frac{\delta_{c b}}{4} G_{\nu}\left(t_{k}\right) \text {. }
$$

Using the chain rule

$$
\begin{gathered}
\frac{\partial}{\partial \mathcal{M}_{a c}^{\dagger}}=\sum_{k \geqslant 1}\left[\frac{\left(\mathcal{M} \mathcal{M}^{\dagger}\right)^{k-1} \mathcal{M}}{4^{k}}\right]_{c a} \partial_{k}, \\
\frac{\partial}{\partial \mathcal{M}_{b a}}=\sum_{k \geqslant 1}\left[\frac{\mathcal{M}^{\dagger}\left(\mathcal{M} \mathcal{M}^{\dagger}\right)^{k-1}}{4^{k}}\right]_{a b} \partial_{k},
\end{gathered}
$$


with $\partial_{k}=\partial / \partial t_{k}$ we immediately obtain, from Eq. (9),

$$
\sum_{s=1}^{\infty}\left(\frac{\mathcal{M} \mathcal{M}^{\dagger}}{4}\right)_{c b}^{s-1}\left[\mathcal{L}_{s}^{\beta=2}-\delta_{s, 1}\right] G_{\nu}\left(t_{k}\right)=0
$$

where

$$
\mathcal{L}_{s}^{\beta=2}=\sum_{k=1}^{s-1} \partial_{k} \partial_{s-k}+\sum_{k \geqslant 1} k t_{k} \partial_{s+k}+\left(N_{f}+\nu\right) \partial_{s}, \quad s \geqslant 1,
$$

and our convention throughout this paper is that terms like the first one in the expression for $\mathcal{L}_{s}$ vanish for $s=1$. The operators $\mathcal{L}_{s}$ obey a sub-algebra of the Virasoro algebra

$$
\left[\mathcal{L}_{r}, \mathcal{L}_{s}\right]=(r-s) \mathcal{L}_{r+s}
$$

without central charge and $r, s \geqslant 1$.

At fixed value $N_{f}$ the $t_{k}$ are not independent, and the coefficients of the expansion (7) are not determined uniquely. ${ }^{1}$ In Eq. (11) the matrix elements of $\mathcal{M M}{ }^{\dagger}$ and the $t_{k}$ are not independent so that we cannot conclude from Eq. (11) that the coefficients of $\left(\mathcal{M M}^{\dagger}\right)^{s}$ vanish. Indeed, the corresponding equations for $N_{f}=1$ and $\nu=0$ are inconsistent. However, in order to fix the coefficients in Eq. (7) uniquely, we may supplement Eq. (11) with additional equations. We do that by requiring that all coefficients of $\left(\mathcal{M} \mathcal{M}^{\dagger}\right)^{s}$ vanish,

$$
\left[\mathcal{L}_{s}^{\beta=2}-\delta_{s, 1}\right] G_{\nu}\left(t_{k}\right)=0, \quad s \geqslant 1 .
$$

This procedure is justified provided that the all equations are consistent. Indeed, for $N_{f} \rightarrow \infty$ the matrix elements and the $t_{k}$ are independent so that Eq. (14) must be valid. By inserting the Taylor expansion (7) into Eq. (14) we obtain an inhomogeneous set of linear equations for the coefficients which depend on the parameter $N_{f}+\nu$. Inconsistencies can only arise for isolated values of $N_{f}+\nu$ for which the homogeneous part of the equations becomes linearly dependent. This is indeed what happens in the example $N_{f}=1$ and $\nu=0$.

Because of the commutation relations (13) the constraints for $s \geqslant 3$ follow from the constraints for $s=1$ and $s=2$. The coefficients of the Taylor expansion (7) can be found recursively by solving the constraints for $s=1$ and $s=2$. This will be carried out in Sec. III after deriving the Virasoro constraints for $\beta=4$ and $\beta=1$.

\section{B. $\beta=4$}

Similarly, we now derive the Virasoro constraints for adjoint fermions $(\beta=4)$. For fermions in the adjoint representation, the zero-momentum Goldstone modes belong to the coset space $S U\left(N_{f}\right) / S O\left(N_{f}\right)$. These Goldstone fields are conveniently parametrized by $U U^{T}$ with $U \in S U\left(N_{f}\right)$. In the sector of topological charge $\nu$, we thus find the finite volume partition function $[10,29]$,

\footnotetext{
${ }^{1}$ Of course, they are determined uniquely up to the order that the $t_{k}$ are independent.
}

$$
\begin{aligned}
\mathcal{Z}_{\frac{\nu}{\nu}}^{\beta} & =4\left(\mathcal{M}_{S}, \mathcal{M}_{S}^{\dagger}\right) \\
& =\int_{U \in U\left(N_{f}\right)} d U(\operatorname{det} U)^{2 \bar{\nu}} e^{(1 / 2) \operatorname{Tr}\left(\mathcal{M}_{S}\left(U U^{t}\right)^{\dagger}+\mathcal{M}_{S}^{\dagger} U U^{t}\right)},
\end{aligned}
$$

where $\bar{\nu}=N_{c} \nu$ and $\mathcal{M}_{S}=M \Sigma V$. In this case the mass matrix $\mathcal{M}_{S}$ is an arbitrary symmetric complex matrix. From $\mathcal{M}_{S}$ $\rightarrow V^{t} \mathcal{M}_{S} V$ we obtain the transformation law

$$
\begin{aligned}
(\operatorname{det} & \left.\mathcal{M}_{S}\right)^{-\bar{\nu}} \mathcal{Z}_{\bar{\nu}}^{\beta=4}\left(\mathcal{M}_{S}, \mathcal{M}_{S}^{\dagger}\right) \\
& =\left(\operatorname{det} V^{t} \mathcal{M}_{S} V\right)^{-\bar{\nu}} \mathcal{Z}_{\bar{\nu}}^{\beta=4}\left(V^{t} \mathcal{M}_{S} V, V^{-1} \mathcal{M}_{S}^{\dagger}\left(V^{t}\right)^{-1}\right) .
\end{aligned}
$$

This implies that $\left(\operatorname{det} \mathcal{M}_{S}\right)^{-\bar{\nu}} \mathcal{Z}_{\bar{\nu}}^{\beta=4}\left(\mathcal{M}_{S}, \mathcal{M}_{S}^{\dagger}\right)$ is a symmetric function of the eigenvalues of $\mathcal{M}_{S} \mathcal{M}_{S}^{\dagger}$. We thus have that

$$
\begin{aligned}
& \left(\operatorname{det} \mathcal{M}_{S}\right)^{-\bar{\nu}} \mathcal{Z}_{\bar{\nu}}^{\beta=4}\left(\mathcal{M}_{S}, \mathcal{M}_{S}^{\dagger}\right)=G_{\nu}^{\beta=4}\left(t_{k}^{S}\right)=1+a_{1}^{S} t_{1}^{S}+a_{2}^{S} t_{2}^{S} \\
& +a_{11}^{S}\left(t_{1}^{S}\right)^{2}+\cdots,
\end{aligned}
$$

where in analogy with Eq. (5) we have defined

$$
t_{k}^{S}=\frac{1}{k} \operatorname{Tr}\left(\frac{\mathcal{M}_{S} \mathcal{M}_{S}^{\dagger}}{4}\right)^{k}
$$

and $G_{\nu}^{\beta=4}\left(t_{k}^{S}\right)$ will be determined by the differential equation

$$
\frac{\partial^{2} \mathcal{Z} \frac{\beta}{\nu}=4}{\partial \mathcal{M}_{S b a} \partial \mathcal{M}_{S a c}^{\dagger}}=\delta_{b c} \mathcal{Z}_{\nu}^{\beta=4}
$$

After substituting the expansion (17) we obtain a differential equation for $G_{\bar{\nu}}^{\beta=4}\left(t_{k}^{S}\right)$ which can be written in the form of the Virasoro constraints,

$$
\left[\mathcal{L}_{s}^{\beta=4}-2 \delta_{s, 1}\right] G_{\nu}^{\beta=4}\left(t_{k}^{S}\right)=0, \quad s \geqslant 1,
$$

where

$$
\mathcal{L}_{s}^{\beta=4}=2 \sum_{k=1}^{s-1} \partial_{k}^{S} \partial_{s-k}^{S}+\sum_{k \geqslant 1} k t_{k}^{S} \partial_{s+k}^{S}+\left(N_{f}+2 \bar{\nu}+s\right) \partial_{s}^{S},
$$

and $\partial_{k}^{S}=\partial / \partial t_{k}^{S}$. One can easily check that $\mathcal{L}_{s}^{\beta=4}$ and $\mathcal{L}_{s}^{\beta=2}$ satisfy the same algebra.

\section{C. $\beta=1$}

For QCD with fundamental fermions and $N_{c}=2$ the Lagrangian can be written in terms of fermion multiplets of length $2 N_{f}$ containing $N_{f}$ quarks and $N_{f}$ anti-quarks. The chiral symmetry is thus enhanced to $S U\left(2 N_{f}\right)$. Since the fermion condensate is anti-symmetric in this enlarged flavor space, the coset space of the Goldstone modes is given by $S U\left(2 N_{f}\right) / S p\left(2 N_{f}\right)$. This coset manifold is parametrized by $U I U^{t}$ where $U \in S U\left(2 N_{f}\right)$ and $I$ is the $2 N_{f} \times 2 N_{f}$ antisymmetric unit matrix 


$$
I=\left(\begin{array}{cc}
0 & \mathbf{1} \\
-\mathbf{1} & 0
\end{array}\right)
$$

The partition function in the sector of fixed topological charge $\nu$ is then given by integrating over the group manifold $U\left(2 N_{f}\right)$ instead of $S U\left(2 N_{f}\right)$,

$$
\begin{aligned}
\mathcal{Z}_{\nu}^{\beta=} & \left.=\tilde{\mathcal{M}}_{A}, \tilde{\mathcal{M}}_{A}^{\dagger}\right) \\
& =\int_{U \in U\left(2 N_{f}\right)} d U(\operatorname{det} U)^{v} e^{(1 / 4) \operatorname{Tr}\left(\tilde{\mathcal{M}}_{A}^{\dagger} U I U^{t}+\tilde{\mathcal{M}}_{A}\left(U I U^{t}\right)^{\dagger}\right)},
\end{aligned}
$$

where the mass matrix $\tilde{\mathcal{M}}_{A}=M V \Sigma$ is an arbitrary antisymmetric complex matrix. In addition to the usual massterm given by

$$
\left(\begin{array}{cc}
0 & \mathcal{M} \\
-\mathcal{M} & 0
\end{array}\right)
$$

it contains di-quark source terms in its diagonal blocks. Below, in the calculation of the mass dependence of the partition function, the di-quark source terms will be put equal to zero. The covariance properties of $\mathcal{Z}_{v}^{\beta=1}$ are given by

$$
\begin{aligned}
& \left(\operatorname{det} \tilde{\mathcal{M}}_{A}\right)^{-\nu / 2} \mathcal{Z}_{\nu}^{\beta=1}\left(\tilde{\mathcal{M}}_{A}, \tilde{\mathcal{M}}_{A}^{\dagger}\right) \\
& \quad=\left(\operatorname{det} V^{t} \tilde{\mathcal{M}}_{A} V\right)^{-\nu / 2} \mathcal{Z}_{\nu}^{\beta=1}\left(V^{t} \tilde{\mathcal{M}}_{A} V, V^{-1} \tilde{\mathcal{M}}_{A}^{\dagger} V^{t-1}\right) .
\end{aligned}
$$

We thus have that $\left(\operatorname{det} \tilde{\mathcal{M}}_{A}\right)^{-\nu / 2} \mathcal{Z}_{\nu}^{\beta=1}\left(\tilde{\mathcal{M}}_{A}, \widetilde{\mathcal{M}}_{A}^{\dagger}\right)$ is a symmetric function of the eigenvalues of $\widetilde{\mathcal{M}}_{A} \widetilde{\mathcal{M}}_{A}^{\dagger}$. This results in the expansion

$$
\begin{aligned}
& \left(\operatorname{det} \tilde{\mathcal{M}}_{A}\right)^{-\nu / 2} \mathcal{Z}_{\nu}^{\beta=1}\left(\tilde{\mathcal{M}}_{A}, \tilde{\mathcal{M}}_{A}^{\dagger}\right) \\
& \quad=G_{\nu}^{\beta=1}\left(t_{k}^{A}\right) \\
& \quad=1+a_{1}^{A} t_{1}^{A}+a_{2}^{A} t_{2}^{A}+a_{11}^{A}\left(t_{1}^{A}\right)^{2}+\cdots,
\end{aligned}
$$

where

$$
t_{k}^{A}=\frac{1}{2 k} \operatorname{Tr}\left(\frac{\tilde{\mathcal{M}}_{A} \tilde{\mathcal{M}}_{A}^{\dagger}}{4}\right)^{k}
$$

The factor $\frac{1}{2}$ in the above definition of $t_{k}^{A}$ takes into account that $\tilde{\mathcal{M}}$ is a $2 N_{f} \times 2 N_{f}$ matrix for $\beta=1$. Substituting Eq. (26) in the differential equation

$$
\frac{\partial^{2} \mathcal{Z}_{\nu=1}^{\beta=1}}{\partial \tilde{\mathcal{M}}_{A b a} \partial \tilde{\mathcal{M}}_{A a c}^{\dagger}}=\frac{\delta_{b c}}{4} \mathcal{Z}_{\nu}^{\beta=1},
$$

we deduce the differential equations for $G_{\nu}^{\beta=1}\left(t_{k}^{A}\right)$ in the form of Virasoro constraints:

$$
\left(\mathcal{L}_{s}^{\beta=1}-\frac{\delta_{s, 1}}{2}\right) G_{v}^{\beta=1}\left(t_{k}^{A}\right)=0,
$$

with

$$
\mathcal{L}_{s}^{\beta=1}=\frac{1}{2} \sum_{k=1}^{s-1} \partial_{k}^{A} \partial_{s-k}^{A}+\sum_{k \geqslant 1} k t_{k}^{A} \partial_{s+k}^{A}+\left(N_{f}+\frac{\nu}{2}-\frac{s}{2}\right) \partial_{s}^{A},
$$

and $\partial_{k}^{A}=\partial / \partial t_{k}^{A}$. The $\mathcal{L}_{s}^{\beta=1}$ satisfy the same algebra as the two other values of $\beta$ discussed in previous sections.

\section{SOLVING THE CONSTRAINTS}

In the first part of this section we discuss the recursive solution of the Virasoro constraints. Flavor-topology duality and nonperturbative solutions are discussed in the second part of this section.

\section{A. Recursive solution of Virasoro constraints}

Remarkably, in all three cases, $\beta=1, \beta=2$ and $\beta=4$, the constraints (14), (20) and (29) can be written in the unified form:

$$
\left(\mathcal{L}_{s}^{\beta}-\frac{1}{\gamma} \delta_{s, 1}\right) G(\alpha, \gamma)=0
$$

where [see Eqs. (12), (21) and (30)]

$$
\begin{aligned}
\mathcal{L}_{s}^{\beta}= & \frac{1}{\gamma} \sum_{k=1}^{s-1} \partial_{k} \partial_{s-k}+\sum_{k \geqslant 1} k t_{k} \partial_{s+k} \\
& +\frac{1}{\gamma}[\alpha+\gamma(2-s)+s-1] \partial_{s},
\end{aligned}
$$

and, motivated by chRMT results of [31], we have introduced the notation

$$
\begin{aligned}
& \alpha=\left(N_{f}-2\right) 2 / \beta+\nu+1, \\
& \gamma=2 / \beta .
\end{aligned}
$$

The $t_{k}$ are defined as in Eqs. (5), (18) and (27) for $\beta$ $=2,4,1$, respectively. From Eq. (31) we conclude that in all three cases the Virasoro constraints only depend on $N_{f}$ and $\nu$ through the combination $N_{f}+\beta \nu / 2(\nu \rightarrow \bar{\nu}$ for $\beta=4)$. Proceeding further, we can recursively solve Eq. (31) by substituting the Taylor expansion of the form (7) for $G(\alpha, \gamma)$ in Eq. (31). By treating the $t_{k}$ as independent variables, we find the following relations between the expansion coefficients:

$$
\begin{aligned}
& \gamma \sum_{k=1}^{\infty} k\left(n_{k+1}+1\right) a_{n_{1} \cdots n_{k}-1 n_{k+1}+1 n_{k+2} \ldots} \\
& +(\alpha+\gamma)\left(n_{1}+1\right) a_{n_{1}+1 n_{2}} \ldots=a_{n_{1} n_{2}} \ldots,
\end{aligned}
$$

for $s=1$ and

$$
\begin{aligned}
& \left(n_{1}+2\right)\left(n_{1}+1\right) a_{n_{1}+2 n_{2}} \ldots+\gamma \sum_{k=1}^{\infty} k\left(n_{k+2}+1\right) \\
& \quad \times a_{n_{1} \cdots n_{k}-1 n_{k+1} n_{k+2}+1 \ldots+(\alpha+1)\left(n_{2}+1\right)} \\
& \quad \times a_{n_{1} n_{2}+1 n_{3} \ldots}=0
\end{aligned}
$$

for $s=2$. (The subscript $n_{1} n_{2} \ldots$ of the coefficients $a_{n_{1} n_{2} \ldots}$ is a shorthand for the partition $1^{n_{1}} 2^{n_{2}} \ldots$.) All higher order Virasoro constraints are satisfied trivially through the Virasoro algebra. If we denote the level of the coefficients by $n \equiv \Sigma_{k} k n_{k}$ and the total number of partitions of $n$ by $p(n)$, the total number of unknown coefficients at 
level $n+2$ is equal to $p(n+2)$ whereas the total number of inhomogeneous equation (for $s=1$ ) is equal $p(n+1)$ and the total number of homogenous equations (for $s=2$ ) is equal to $p(n)$. The total number of partitions satisfies the recursion relation [30]

$$
\begin{aligned}
p(n+2)= & p(n+1)+p(n)-p(n-3)-p(n-5)+\cdots \\
& -(-1)^{k} p\left(n+2-\frac{3 k^{2}-k}{2}\right)-(-1)^{k} p(n+2 \\
& \left.-\frac{3 k^{2}+k}{2}\right)+\cdots
\end{aligned}
$$

Since the number of partitions is a monotonic function of $n$, we have that

$$
p(n+2) \leqslant p(n+1)+p(n),
$$

and the number of equations is always larger than or equal to the number of coefficients. In Table I we give the total number of coefficients and the total number of equations up to level $n=10$.

From normalization condition $a_{0}=1$ we conclude that $a_{1}=1 /(\alpha+\gamma)$. At level $n=2$ we obtain one equation each from Eqs. (34) and (35), respectively,
TABLE I. The total number of unknown coefficients $[P(n)]$ and the total number of equations $[P(n-1)+P(n-2)]$ at level $n$.

\begin{tabular}{cccccc}
\hline \hline$n$ & $P(n)$ & $P(n-1)+P(n-2)$ & $n$ & $P(n)$ & $P(n-1)+P(n-2)$ \\
\hline 1 & 1 & 1 & 6 & 11 & 12 \\
2 & 2 & 2 & 7 & 15 & 18 \\
3 & 3 & 3 & 8 & 22 & 26 \\
4 & 5 & 5 & 9 & 30 & 37 \\
5 & 7 & 8 & 10 & 42 & 52 \\
\hline \hline
\end{tabular}

$$
(\alpha+1) a_{2}+2 a_{11}=0
$$$$
\gamma a_{2}+2(\alpha+\gamma) a_{11}=a_{1}=1 /(\alpha+\gamma),
$$

which are solved by

$$
\begin{aligned}
a_{11} & =\frac{(1+\alpha)}{2 \alpha(\alpha+\gamma)(\alpha+\gamma+1)} \\
a_{2} & =\frac{-1}{\alpha(\alpha+\gamma)(\alpha+\gamma+1)}
\end{aligned}
$$

Up to order $\left(\mathcal{M} \mathcal{M}^{\dagger}\right)^{4}$ we find

$$
\begin{aligned}
& \mathcal{Z}_{\nu, N_{f}}^{\beta}\left(\mathcal{M}, \mathcal{M}^{\dagger}\right)=(\operatorname{det} \mathcal{M})^{\nu}\left[1+\frac{\operatorname{Tr}\left(\mathcal{M} \mathcal{M}^{\dagger}\right)}{4(\alpha+\gamma)}+\frac{(\alpha+1)\left(\operatorname{Tr} \mathcal{M} \mathcal{M}^{\dagger}\right)^{2}}{32 \alpha(\alpha+\gamma)(\alpha+\gamma+1)}-\frac{\operatorname{Tr}\left(\mathcal{M} \mathcal{M}^{\dagger}\right)^{2}}{32 \alpha(\alpha+\gamma)(\alpha+\gamma+1)}\right. \\
& +\frac{2 \operatorname{Tr}\left(\mathcal{M} \mathcal{M}^{\dagger}\right)^{3}}{4^{3} 3 \alpha\left(\alpha^{2}-\gamma^{2}\right)(\alpha+\gamma+1)(\alpha+\gamma+2)}-\frac{(\alpha-\gamma+2) \operatorname{Tr}\left(\mathcal{M} \mathcal{M}^{\dagger}\right) \operatorname{Tr}\left(\mathcal{M} \mathcal{M}^{\dagger}\right)^{2}}{4^{3} 2 \alpha\left(\alpha^{2}-\gamma^{2}\right)(\alpha+\gamma+1)(\alpha+\gamma+2)} \\
& +\frac{[(\alpha-\gamma)(\alpha+3)+2]\left[\operatorname{Tr}\left(\mathcal{M M}^{\dagger}\right)\right]^{3}}{4^{3} 6 \alpha\left(\alpha^{2}-\gamma^{2}\right)(\alpha+\gamma+1)(\alpha+\gamma+2)} \\
& +\frac{(\gamma-5 \alpha-6) \operatorname{Tr}\left(\mathcal{M M}^{\dagger}\right)^{4}}{4^{5} \alpha(\alpha+1)\left(\alpha^{2}-\gamma^{2}\right)(\alpha+\gamma+1)(\alpha+\gamma+2)(\alpha+\gamma+3)(\alpha-2 \gamma)} \\
& -\frac{\left[2 \alpha^{2}-4 \alpha(\gamma-2)-7 \gamma+6\right] \operatorname{Tr}\left(\mathcal{M M}^{\dagger}\right) \operatorname{Tr}\left(\mathcal{M} \mathcal{M}^{\dagger}\right)^{3}}{4^{4} 3 \alpha(\alpha+1)\left(\alpha^{2}-\gamma^{2}\right)(\alpha+\gamma+1)(\alpha+\gamma+2)(\alpha+\gamma+3)(\alpha-2 \gamma)} \\
& +\frac{\left[\alpha^{2}+\alpha(5-3 \gamma)+2 \gamma^{2}-\gamma+6\right]\left[\operatorname{Tr}\left(\mathcal{M} \mathcal{M}^{\dagger}\right)^{2}\right]^{2}}{4^{5} 2 \alpha(\alpha+1)\left(\alpha^{2}-\gamma^{2}\right)(\alpha+\gamma+1)(\alpha+\gamma+2)(\alpha+\gamma+3)(\alpha-2 \gamma)} \\
& +\frac{\left[3 \alpha^{2}(\gamma-2)-\alpha^{3}-6-4 \gamma^{2}+13 \gamma+\alpha\left(-11+14 \gamma-2 \gamma^{2}\right)\right]\left(\operatorname{Tr} \mathcal{M} \mathcal{M}^{\dagger}\right)^{2} \operatorname{Tr}\left(\mathcal{M M}^{\dagger}\right)^{2}}{4^{5} \alpha(\alpha+1)\left(\alpha^{2}-\gamma^{2}\right)(\alpha+\gamma+1)(\alpha+\gamma+2)(\alpha+\gamma+3)(\alpha-2 \gamma)} \\
& +\frac{\left[6+\alpha^{4}+\alpha^{3}(7-3 \gamma)-25 \gamma+18 \gamma^{2}+\alpha^{2}\left(17-21 \gamma+2 \gamma^{2}\right)+\alpha\left(17-43 \gamma+14 \gamma^{2}\right)\right]\left(\operatorname{Tr} \mathcal{M M}^{\dagger}\right)^{4}}{4^{5} 6 \alpha(\alpha+1)\left(\alpha^{2}-\gamma^{2}\right)(\alpha+\gamma+1)(\alpha+\gamma+2)(\alpha+\gamma+3)(\alpha-2 \gamma)} \\
& \left.+\mathcal{O}\left(\left(\mathcal{M M}^{\dagger}\right)^{6}\right)\right] \text {. }
\end{aligned}
$$


For $\beta=1$ the mass matrix $\tilde{\mathcal{M}}$ has been expressed in terms of the standard $N_{f} \times N_{f}$ mass matrix that occurs in the QCD partition function [see Eq. (24)]. For $\beta=2$ and $\beta=4$ the matrix $\mathcal{M}$ is a complex $N_{f} \times N_{f}$ matrix as well, but for $\beta$ $=4$ it is symmetric. We have checked that all coefficients in Eq. (40) agree with the corresponding expansion for $\beta=2$ obtained in [20]. When the masses are degenerate $\left(\mathcal{M M}^{\dagger}\right)_{a b}=\mu^{2} \delta_{a b}$ exact expressions for $\mathcal{Z}_{\nu, N_{f}}^{\beta}$ are known for all three values of the Dyson index. For instance, in the special case $N_{f}=2$ and $\beta=1$ the finite volume partition function is given by [29]

$$
\mathcal{Z}_{\nu, 2}^{\beta=1}=2 \sum_{k=0}^{\infty}\left(\frac{\mu^{2}}{4}\right)^{(\nu+k)} \frac{[2(\nu+k)] !}{k !(\nu+k) !(\nu+k+2) !(2 \nu+k) !},
$$

which is a convergent series for $\mathcal{Z}_{\nu, 2}^{\beta=1}$. The reader can check that Eq. (40) only differs from Eq. (41) by the overall normalization factor $2^{1-2 \nu} /(\nu !(\nu+2) !)$. We have also made similar checks for $\beta=4$. The poles that appear in the expansion (40) at finite $N_{f}$, the so called de Wit-'t Hooft poles [32], cancel after rewriting the expansion in powers of products of the eigenvalues of $\mathcal{M}^{\dagger} \mathcal{M}$. They can be regulated by choosing a noninteger value for the topological charge $[20,33]$.

The expansion in terms of traces of the quark mass matrix can be directly compared with $\left\langle\Pi_{a=1}^{N_{f}} \operatorname{det}\left(D+m_{a}\right)\right\rangle_{\text {Yang-Mills }}$ from which we derive spectral sum-rules for inverse powers of the eigenvalues of the Dirac operator [10]. With $\zeta_{k}$ related to the eigenvalues $\pm i \lambda_{k}$ of the Dirac operator through $\zeta_{k}$ $=V \Sigma \lambda_{k}$, the first six sum rules for $m_{a}=0$ are given by

$$
\begin{aligned}
\left\langle\sum_{n} \frac{1}{\zeta_{n}^{2}}\right\rangle & =\frac{1}{4(\alpha+\gamma)}, \\
\left\langle\sum_{n} \frac{1}{\zeta_{n}^{4}}\right\rangle & =\frac{1}{16 \alpha(\alpha+\gamma)(\alpha+\gamma+1)} \\
\left\langle\left(\sum_{n} \frac{1}{\zeta_{n}^{2}}\right)^{2}\right\rangle & =\frac{\alpha+1}{16 \alpha(\alpha+\gamma)(\alpha+\gamma+1)} \\
\left\langle\sum_{n} \frac{1}{\zeta_{n}^{6}}\right\rangle & =\frac{1}{32 \alpha\left(\alpha^{2}-\gamma^{2}\right)(\alpha+\gamma+1)(\alpha+\gamma+2)} \\
\left\langle\sum_{n} \frac{1}{\zeta_{n}^{2}} \sum_{m} \frac{1}{\zeta_{m}^{4}}\right\rangle & =\frac{\alpha-\gamma+2}{64 \alpha\left(\alpha^{2}-\gamma^{2}\right)(\alpha+\gamma+1)(\alpha+\gamma+2)} \\
\left\langle\left(\sum_{n} \frac{1}{\zeta_{n}^{2}}\right)^{3}\right\rangle & =\frac{(\alpha-\gamma)(\alpha+3)+2}{64 \alpha\left(\alpha^{2}-\gamma^{2}\right)(\alpha+\gamma+1)(\alpha+\gamma+2)}
\end{aligned}
$$

The sum is over the positive eigenvalues only. It is clear that all sum rules will depend on the topological charge through the combination $N_{f}+\beta \nu / 2$. The first formula in Eq. (42) had already been obtained in [29] from the low-energy QCD fi- nite volume partition functions. For $\beta=2$ our sum rules agree with the ones derived in [20] obtained by means of Virasoro constraints and flavor-topology duality. The remaining sum rules have never been derived before from the finite volume partition functions for $\beta=1,4$, but all except the fourth one have been obtained from chRMT by means of Selberg integrals for arbitrary $\beta$ [31].

\section{B. Flavor-topology duality}

So far for the sum rules, we now return to the flavortopology duality relations which is our main result. To this end we construct a $\left(N_{f}+\beta \nu / 2\right) \times\left(N_{f}+\beta \nu / 2\right)$ matrix $\overline{\mathcal{M}}$ from the $N_{f} \times N_{f}$ original mass-matrix $\mathcal{M}$ by adding zeros. We thus have that $\operatorname{Tr}\left(\mathcal{M} \mathcal{M}^{\dagger}\right)^{k}=\operatorname{Tr}\left(\overline{\mathcal{M}} \overline{\mathcal{M}}^{\dagger}\right)^{k}$. The flavortopology duality relation then follows from the observation that all coefficients in the small-mass expansion (40) depend on the number of flavors explicitly through the combination $N_{f}+\beta \nu / 2$ and can be written as

$$
\mathcal{Z}_{\nu, N_{f}}^{\beta}\left(\mathcal{M}, \mathcal{M}^{\dagger}\right)=(\operatorname{det} \mathcal{M})^{\nu} \mathcal{Z}_{0, N_{f}+\beta \nu / 2}^{\beta}\left(\overline{\mathcal{M}}, \overline{\mathcal{M}}^{\dagger}\right),
$$

where $\nu \rightarrow \bar{\nu}$ for $\beta=4$. For the cases where $\beta \nu / 2$ is not an integer, the flavor-topology duality should be understood as an analytical continuation ${ }^{2}$ in $\nu$. Strictly speaking, we have only shown the duality relation (43) for the recursion relations for the coefficients of the small-mass expansion of the partition functions. To complete our proof we have to show that the Virasoro constraints uniquely determine all coefficients and that the expansion is a convergent series. The convergence of the small mass expansion follows immediately from the compactness of the unitary group. Indeed, for $\beta=2$, the small-mass expansion can be resummed to the $\tau$-function of a KP hierarchy $[19,16]$. This result can also be obtained from an exact reconstruction of $\mathcal{Z}_{v}^{\beta=2}$ [28] from the character expansion [27] which is an alternative to the smallmass expansion derived here. The uniqueness of the solution of the Virasoro constraints is a much more difficult question [34]. We have checked explicitly to the order $\left(\mathcal{M M}^{\dagger}\right)^{8}$ that this is indeed the case for all values of the parameters.

For $\beta=2$, however, the uniqueness follows from the explicit solution [35] of the equation

$$
\frac{\partial^{2}}{\partial \mathcal{M}_{b a} \partial \mathcal{M}_{a b}^{\dagger}} \mathcal{Z}_{\nu}^{\beta=2}\left(\mathcal{M}, \mathcal{M}^{\dagger}\right)=\frac{N_{f}}{4} \mathcal{Z}_{\nu}^{\beta=2}\left(\mathcal{M}, \mathcal{M}^{\dagger}\right)
$$

obtained from the trace of Eq. (8) or the Schwinger-Dyson equation [21]

\footnotetext{
${ }^{2}$ The analytical continuation in $\nu$ is subtle. The correct continuation is obtained by $[26,12]$ expressing the finite volume partition function in terms of modified Bessel functions and make an analytical continuation in the index of the Bessel functions.
} 


$$
\begin{gathered}
\mathcal{M}_{c d}^{\dagger} \mathcal{M}_{d b} \frac{\partial^{2}}{\partial \mathcal{M}_{b a} \partial \mathcal{M}_{a c}^{\dagger}} \mathcal{Z}_{\nu}^{\beta=2}\left(\mathcal{M}, \mathcal{M}^{\dagger}\right) \\
=\mathcal{M}_{b a}^{\dagger} \mathcal{M}_{a b} \frac{N_{f}}{4} \mathcal{Z}_{\nu}^{\beta=2}\left(\mathcal{M}, \mathcal{M}^{\dagger}\right)
\end{gathered}
$$

obtained by contracting Eq. (8) with $\mathcal{M}$ and $\mathcal{M}^{\dagger}$. In both cases, the invariance of the partition function can be used to reduce the equations to a separable differential equation in the eigenvalues of $\mathcal{M}^{\dagger} \mathcal{M}$. Because we are looking for a solution that is symmetric in the eigenvalues satisfying the boundary condition $G_{\nu}(0)=1$, it is determined uniquely by the solution of the single particle equation which happens to be the Bessel equation.

Let us discuss this in more detail for Eq. (44). The first step is to write the partition function as

$$
\mathcal{Z}_{\nu}^{\beta=2}\left(\mathcal{M}, \mathcal{M}^{\dagger}\right)=\left(\frac{\operatorname{det} \mathcal{M}}{\operatorname{det} \mathcal{M}^{\dagger}}\right)^{\nu / 2} \widetilde{\mathcal{Z}}_{\nu}(\Lambda),
$$

where $\widetilde{\mathcal{Z}}_{\nu}(\Lambda)$ is a function of the eigenvalues $x_{k}$ of $\mathcal{M}^{\dagger} \mathcal{M}$ only $\left[\Lambda=\operatorname{diag}\left(x_{1}, \ldots x_{N_{f}}\right)\right]$. It satisfies the differential equation

$$
\left[\frac{\partial^{2}}{\partial \mathcal{M}_{b a} \partial \mathcal{M}_{a b}^{\dagger}}-\sum_{k} \frac{\nu^{2}}{4 x_{k}^{2}}\right] \widetilde{\mathcal{Z}}_{\nu}(\Lambda)=\frac{N_{f}}{4} \widetilde{\mathcal{Z}}_{\nu}(\Lambda) .
$$

This equation can be further simplified by introducing the reduced partition function

$$
z_{\nu}(\Lambda)=\Delta\left(x_{k}^{2}\right) \prod_{k} \sqrt{x}_{k} \widetilde{\mathcal{Z}}_{\nu}(\Lambda)
$$

where the Vandermonde determinant is defined by $\Delta\left(x_{k}^{2}\right)$ $=\Pi_{k<l}\left(x_{k}^{2}-x_{l}^{2}\right)$. We thus have that $z_{\nu}(\Lambda)$ is a completely antisymmetric function of the eigenvalues with boundary condition such that $\widetilde{\mathcal{Z}}_{\nu}(\Lambda) \sim \prod_{k} x_{k}^{\nu}$ for $x_{k} \rightarrow 0$. The reduced partition function satisfies the separable differential equation

$$
\sum_{k}\left[\partial_{k}^{2}-\frac{4 \nu^{2}-1}{4 x_{k}^{2}}\right] z_{\nu}(\Lambda)=N_{f} z_{\nu}(\Lambda) .
$$

The solution can thus be expressed as a Slater-determinant

$$
z_{\nu}(\Lambda)=\operatorname{det}_{k l}\left[\phi_{k}\left(x_{l}\right)\right]
$$

where the $\phi_{k}$ are the regular solutions of the single particle equation up to terms that vanish in the determinant. This requires further discussion. The solution of the single particle equation

$$
D_{B} \phi_{k}(x) \equiv\left[\partial_{x}^{2}-\frac{4 \nu^{2}-1}{4 x^{2}}\right] \phi_{k}(x)=\omega_{k}^{2} \phi_{k}(x),
$$

is the modified Bessel function

$$
\phi_{k}(x)=\sqrt{x} I_{\nu}\left(\omega_{k} x\right)
$$

In order to cancel the Vandermonde determinant for $x_{k} \rightarrow 0$ we necessarily have that all $\omega_{k}^{2}$ are equal and thus $\omega_{k}=1$. Because of the antisymmetry of the columns in the Slater determinant (50) we can allow for single particle solutions that satisfy the equations

$$
\begin{aligned}
& D_{B} \phi_{1}(x)=\phi_{1}(x), \\
& D_{B} \phi_{2}(x)=\phi_{2}(x)+\mu_{21} \phi_{1}(x), \\
& D_{B} \phi_{3}(x)=\phi_{3}(x)+\mu_{31} \phi_{1}(x)+\mu_{32} \phi_{2}(x), \text { etc. }
\end{aligned}
$$

By redefining $\phi_{3}, \phi_{4}, \ldots$ we obtain the equations (for $k$ $\geqslant 2$ )

$$
D_{B} \phi_{k}(x)=\phi_{k}(x)+\mu_{k k-1} \phi_{k-1}(x),
$$

where the $\mu_{k k-1} \phi_{k-1}(x)$ cancel in the expression for the Slater determinant. The solution of these equations follows immediately from the recursion relation

$$
D_{B}\left[x^{k+1 / 2} I_{\nu+k}(x)\right]=x^{k+1 / 2} I_{\nu+k}(x)+2 k x^{k-1 / 2} I_{\nu+k-1}(x) .
$$

We thus find that

$$
\phi_{k}=x^{k+1 / 2} I_{\nu+k}(x)
$$

and $\mu_{k-1}=2 k$. Since the Bessel equation has only one regular solution, we conclude that this solution is unique. The Virasoro constraints together with the boundary conditions determine the partition function uniquely and is given by a Slater-determinant of modified Bessel functions (50) which agrees with the known result for the one-link intergral $[21,19,10,35]$.

Actually the solution of the Virasoro constraints, although not its uniqueness can be obtained in a simpler way by identifying them with constraints which are satisfied by the generalized Kontsevich model (GKM). By changing variables from the matrix elements of $\mathcal{M}$ and $\mathcal{M}^{\dagger}$ to the matrix elements of the Hermitian matrix $L \equiv\left(\mathcal{M M}^{\dagger} / 4\right)$ we can rewrite Eq. (9) as

$$
\left[\frac{\partial}{\partial L_{b e}} L_{d e} \frac{\partial}{\partial L_{d c}}+\nu \frac{\partial}{\partial L_{b c}}\right] G_{\nu}(L)=\delta_{b c} G_{\nu}(L) .
$$

This equation admits a solution in the form of a GKM with potential $\mathcal{V}(X)=1 / X$ where $X$ is a $N_{f} \times N_{f}$ normal matrix. More explicitly we have ${ }^{3}$

$$
G_{\nu}(L)=\int \frac{d X}{(\operatorname{det} X)^{N_{f}-\nu}} e^{\operatorname{Tr}[L X+1 / X]},
$$

which is a simple modification of the $\nu=0$ case studied in [19]. In order to verify that Eq. (58) is a solution of Eq. (57)

\footnotetext{
${ }^{3}$ The contour of integration is such that the integral converges for all values of $L$, and that an integral over a total derivative of $X$ vanishes.
} 
we have to insert a total derivative in the integral over $X$ and multiply it by a second order differential operator as follows:

$$
\frac{\partial}{\partial L_{b e}} \frac{\partial}{\partial L_{d c}} \int d X \frac{\partial}{\partial X_{e d}}\left(\frac{1}{(\operatorname{det} X)^{N_{f}-\nu}} e^{\operatorname{Tr}[L X+1 / X]}\right)=0 .
$$

Concluding, for $\beta=2$ the Virasoro constraints naturally lead to a simple and rigorous proof of flavor-topology duality (43) which is an alternative to the proof presented in $[11,12]$. For $\beta=1$ and $\beta=4$ a direct solution of the Virasoro constraints is not known, although we believe that further progress can be achieved at least in the case of equal quark masses where exact formulas for the partition function are known [29]. For non-degenerate masses the obstacle is the lack of a generalization of the Itzykson-Zuber formula for integrals over symmetric and anti-symmetric unitary matrices. Therefore our proof of flavor-topology duality for $\beta=1$ and $\beta=4$ is only valid under the assumption of the uniqueness of the solution of the Virasoro constraints.

\section{CONCLUSIONS}

In a parameter range where the main contribution to the QCD partition function comes from the zero-momentum component of the Goldstone modes, the partition functions reduce to zero-dimensional integrals over the group manifolds $S U\left(N_{f}\right), S U\left(N_{f}\right) / O\left(N_{f}\right)$ and $S U\left(2 N_{f}\right) / S p\left(2 N_{f}\right)$, for fermions in the fundamental representation and $N_{c} \geqslant 3$ ( $\beta$ $=2), N_{c}=2(\beta=1)$, and adjoint fermions with $N_{c} \geqslant 2(\beta$ $=4)$, respectively. In the sector of fixed topological charge $\nu$ we have shown that these partition functions satisfy differential equations in the form of Virasoro constraints which determine the small-mass expansion for arbitrary values of the topological charge. We have calculated the coefficients of the small mass expansion recursively up to the order $m^{8}$ and have obtained new spectral sum rules for inverse powers of the eigenvalues of the Dirac operator for $\beta=1, \beta=2$ and $\beta=4$. For all three values of $\beta$ the constraints depend on the topological charge through the combination $N_{f}+\beta \nu / 2$ which demonstrates flavor-topology duality for the corresponding small-mass $(m \rightarrow 0)$ expansions. For the special case of $\beta$
$=2$ the uniqueness of the solution of the Virasoro constraints and the convergence of the small mass expansion implies flavor-topology duality for the full (finite $m$ ) partition function. In this case, we have found a non-perturbative solution of the Virasoro constraints in the form of a generalized Kontsevich model for arbitrary topological charge $\nu$ which complements the $\nu=0$ results of [19]. We have not been able to find an analogous solution for $\beta=1$ and $\beta=4$. Based on numerical evidence we believe that for these two cases the solution of the Virasoro constraints is unique as well, but a rigorous proof could not be given. The existence of exact expressions for partition functions with degenerate masses $\left(\mathcal{M M}^{\dagger}\right)_{a b}=\mu^{2} \delta_{a b}$ indicates that further progress is possible.

It is worth commenting that, even for $\beta=2$, although order by order the character expansion is equivalent to the perturbative solution of the small-mass Virasoro constraints, it does not provide us with a natural proof of flavor-topology duality (see [28]). It should also be pointed out that the GKM are known to satisfy both small-mass and large-mass Virasoro constraints. Thus, in view of our results, we also expect to find large-mass constraints for $\nu \neq 0$ at least for $\beta=2$. Indeed we have explicitly obtained such constraints entirely within the context of unitary integrals and they depend quadratically on the topological charge $\nu$, contrary to the linear dependence of the small-mass constraints. The generalization of the large-mass constraints to $\beta=1$ and $\beta=4$ seems to be within reach. Due to the absence of an explicit $N_{f}$ dependence of the expansion coefficients they are the most relevant ones from the point of view of both the replica limit $[20,26,12]$ and the classification of universality classes [36]. In particular, for applications in 2D gravity they correspond to the continuum Virasoro constraints which appear after the double scaling limit.

\section{ACKNOWLEDGMENTS}

This work was partially supported by the U.S. DOE grant DE-FG-88ER40388. The work of D.D. is supported by FAPESP (Brazilian Agency). Poul Damgaard, Kim Splittorff and Dominique Toublan are acknowledged for useful discussions.
[1] G. 't Hooft, Phys. Rev. Lett. 37, 8 (1976); Phys. Rev. D 14, 3432 (1976).

[2] E. P. Wigner, Ann. Math. 53, 36 (1951).

[3] E. V. Shuryak and J. J. M. Verbaarschot, Nucl. Phys. A560, 306 (1993).

[4] J. J. M. Verbaarschot, Phys. Rev. Lett. 72, 2531 (1994).

[5] G. Akemann, P. H. Damgaard, U. Magnea, and S. Nishigaki, Nucl. Phys. B487, 721 (1997).

[6] F. J. Dyson, J. Math. Phys. 3, 1199 (1962).

[7] J. J. M. Verbaarschot, Nucl. Phys. B427, 534 (1994).

[8] J. Verbaarschot, in lectures given at "NATO Advanced Study Institute on Confinement, Duality and Nonperturbative Aspects of QCD," Cambridge, England, 1997, edited by P. van Baal, pp. 343, hep-th/9710114.
[9] J. Gasser and H. Leutwyler, Phys. Lett. B 188, 477 (1987).

[10] H. Leutwyler and A. Smilga, Phys. Rev. D 46, 5607 (1992).

[11] T. Akuzawa and M. Wadati, J. Phys. Soc. Jpn. 67, 2151 (1998).

[12] G. Akemann, D. Dalmazi, P. H. Damgaard, and J. J. M. Verbaarschot, Nucl. Phys. B601, 77 (2001).

[13] P. H. Damgaard, Phys. Lett. B 424, 322 (1998).

[14] G. Akemann and P. H. Damgaard, Phys. Lett. B 432, 390 (1998).

[15] G. Akemann and P. H. Damgaard, Nucl. Phys. B528, 411 (1998).

[16] G. Akemann and P. H. Damgaard, Nucl. Phys. B576, 597 (2000).

[17] P. Rossi, M. Campostrini, and E. Viccari, Phys. Rep. 102, 143 (1998). 
[18] D. Gross and E. Witten, Phys. Rev. D 21, 446 (1980); E. Brézin and D. Gross, Phys. Lett. 97B, 120 (1980).

[19] A. Mironov, A. Morozov, and G. Semenoff, Int. J. Mod. Phys. A 11, 5031 (1996).

[20] P. H. Damgaard and K. Splittorff, Nucl. Phys. B572, 478 (2000); P. H. Damgaard, Phys. Lett. B 476, 465 (2000).

[21] R. C. Brower, P. Rossi, and C.-I. Tan, Nucl. Phys. B190, 699 (1981); Phys. Rev. D 23, 942 (1981); R. C. Brower and M. Nauenberg, Nucl. Phys. B180, 221 (1981).

[22] D. J. Gross and M. Newmann, Nucl. Phys. B380, 168 (1992).

[23] R. Dijkgraaf, in lectures given at the "Cargese Summer School on New Symmetry Principles in Quantum Field Theory," Cargese, 1991, hep-th/9201003.

[24] P. Di Francesco, P. Ginsparg, and J. Zinn-Justin, Phys. Rep. 254, 1 (1995).

[25] E. Abdalla, M. C. B. Abdalla, D. Dalmazi, and A. Zadra, 2DGravity in Non-Critical Strings, Lecture Notes in Physics Vol. m20 (Springer, Berlin, 1994).

[26] D. Dalmazi and J. J. M. Verbaarschot, Nucl. Phys. B592, 419 (2001).

[27] I. Bars, J. Math. Phys. 21, 2678 (1980).

[28] A. B. Balantekin, Phys. Rev. D 62, 085017 (2000).

[29] A. Smilga and J. J. M. Verbaarschot, Phys. Rev. D 51, 829 (1995).

[30] M. Hall, Combinatorial Theory (Blaisdell, Waltham, 1967).

[31] J. J. M. Verbaarschot, Phys. Lett. B 329, 351 (1994).

[32] B. de Wit and G. 't Hooft, Phys. Lett. 69B, 61 (1977).

[33] P. H. Damgaard (private communication).

[34] S. Kharchev, A. Marshakov, A. Mironov, A. Morozov, and A. Zabrodin, Phys. Lett. B 275, 311 (1992).

[35] A. D. Jackson, M. K. Sener, and J. J. M. Verbaarschot, Phys. Lett. B 387, 355 (1996).

[36] R. Dijkgraaf, H. Verlinde, and E. Verlinde, Nucl. Phys. B348, 435 (1991). 